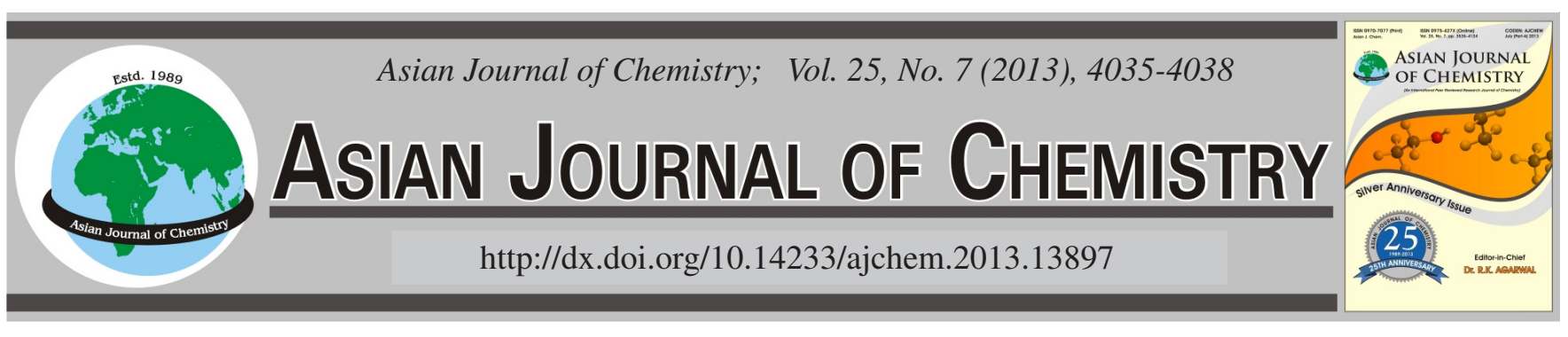

\title{
Fabrication of Platinum Nanowires via Electrodeposition of Platinum-Phenothiazine Complex in freshly prepared Anodic Aluminium Oxide Template
}

\author{
Waleed Ali Mahmmood ${ }^{1}$, Abdulkareem Mohammed Ali Alsammarraie ${ }^{2, *}$ and Alya'a Jabar Ahmed ${ }^{1}$
}

${ }^{1}$ Department of Chemistry, College of Science for Women, Baghdad University, Baghdad, Iraq

${ }^{2}$ Department of Chemistry, College of Science, Baghdad University Jadiriyah, Baghdad, Iraq

*Corresponding author: Tel: +964 7901350456; E-mail: samuraee2000@ hotmail.com

(Received: 12 April 2012;

Accepted: 25 January 2013)

AJC-12748

\begin{abstract}
Porous anodic aluminum oxide film with self-ordered character, has been widely used as the template for fabricating nano-structural materials. In this study, highly ordered anodic aluminum oxide template was fabricated in phosphoric acid by the two-step anodic oxidation process. The anodizing voltage was maintained at $60 \mathrm{~V}$. The results showed that $20 \%$ phosphoric acid and $5{ }^{\circ} \mathrm{C}$ are suitable to prepare anodic aluminum oxide templates with a pore diameters of $130 \mathrm{~nm}$. The fabricated anodic aluminum oxide films then subjected to post electrodepositing of Pt nanowires from freshly prepared Pt-phenothiazine complex, a forest of nanowires with a diameter nearly closed to the started anodic aluminum oxide pores sizes achieved. The morphology and surface structure of the anodic aluminum oxide films were examined before and after electrodepositing by; scanning electron microscopy, atomic force microscope and X-ray diffraction spectroscopy.
\end{abstract}

Key Words: Anodic aluminum oxide template, Platinum nanowires, Phenothiazine complexes, Pt-electrodeposition.

ᄂ - - - - - - - - - - - - - - - - - - - - - - - - -

\section{INTRODUCTION}

Anodization of aluminum has become one of the most popular processing ways used for formation of porous structures with a pore diameter ranging from about 10 to over $300 \mathrm{~nm}^{1}$. In recent years, PAA has generated interest as a candidate material for nanotechnology applications. The pore diameter, pore spacing and film thickness are all controllable through the anodization conditions, such as the choice of electrolyte, temperature and anodization time. The pore spacing may also be controlled through post anodization techniques such as chemical etching ${ }^{2}$. Anodic aluminum oxide nanotubes have been extensively used as a template for the fabrication of nanowires and nanotubes as it possesses many desirable characteristics, including tunable pore dimensions, good mechanical strength and thermal stability. It is used to prepare self assembled arrays of a variety materials, metals, alloys and multilayers that can be incorporated into chemical sensors, solar cells and magneto-optic recording media ${ }^{3}$. While a range of well-developed deposition techniques have already been used to fabricate nano structures (e.g. electrochemical deposition $^{4}$, electroless deposition ${ }^{5}$, chemical vapour deposition ${ }^{6}$ ). Electrochemical method extensively used to deposit metallic materials into nanopores of the templates in order to fabricate one dimensional nanostructures of wires and tubes ${ }^{7-9}$. We have fabricated and tuned properties of platinum nanowires by electrodepositing in the pores of anodic aluminum oxide templates.

\section{EXPERIMENTAL}

\section{Experimental methodology includes two parts:}

Synthesis of anodic aluminium oxide: High-purity aluminum foil $(99.98 \%)$ with a thickness of $0.2 \mathrm{~mm}$ was cut into $32 \mathrm{~mm}$ in diameter circle were employed in present experiment to fabricate the anodic aluminum oxide template. The aluminium foil was first annealed at $500{ }^{\circ} \mathrm{C}$ for $2 \mathrm{~h}$, degreased using acetone and ethanol, then rinsed in distilled water. Aluminium foil was electropolished in a mixed solution of $\mathrm{HClO}_{4}: \mathrm{C}_{2} \mathrm{H}_{5} \mathrm{OH}=1: 4(\mathrm{v} / \mathrm{v})$ for 5 min to provide a smooth surface, then promptly rinsed with distilled water. The foils were then anodized in a homemade Teflon cell, where the aluminum was configured as anode and Pt foil as the cathode using a two-step oxidation process. in this work were fabricated using a $60 \mathrm{~V}$ DC potential with phosphoric acid (20\%) as the electrolyte at 5 for $30 \mathrm{~min}$. The anodized alumina was subsequently dissolved in an etchant solution consisting of 6 wt \% phosphoric acid and $1.8 \mathrm{wt} \%$ chromic acid, followed by a second oxidation step under the same conditions for another $2 \mathrm{~h}$. Channels were finally cleared by dipping the templates into a $5 \mathrm{wt}$. $\%$ phosphoric acid solution at $20^{\circ} \mathrm{C}$ for $10 \mathrm{~min}$. 
Fabrication of platinum nanowire arrays: Platinumphenothiazine complex under investigated was synthesized as follows: The ligand dissolved in $(15 \mathrm{~mL})$ of dimethyl formamide (DMF) followed by addition drop by drop $(5 \mathrm{~mL})$ of metal salt $\left(\mathrm{H}_{2} \mathrm{PtCl}_{6}\right)$. The resulting formation complex having dark brown colour. Electrodeposition of Pt nanowires was carried out at room temperature on the anodic aluminum oxide without removing its back (cathode) the Pt foil served as an anode and freshly prepared Pt-phenothiozine complex as electrolytic solutions. The pore size of the anodic aluminum oxide membrane and the platinum nanowires were examined using a scanning electron microscope (SEM, 54700, Hitachi) and atomic force microscope (AFM, AA 3000, angstrom). The crystallographic structures of the samples were identified using an X-ray diffractometer (XRD- 6000 with $\mathrm{CuK}_{\alpha}$-radiation, wave $1.5 \mathrm{~A}, 40 \mathrm{kV}, 30 \mathrm{~mA}$ ) at a scanning step size of $2 \theta=$ $0.05^{\circ}$ in the $2 \theta$ range from $20^{\circ}$ to $60^{\circ}$. Identification and study of this complex was carried out by percentage of metal analysis using AA-680 Shimadzu atomic absorption spectrophotometer. The electronic spectra of the prepared complex was recorded on a Shimadzu UV-160 spectrophotometer. FTIR spectra of the sample was recorded using IR prestige-21 spectrophotometer as CsI discs.

\section{RESULTS AND DISCUSSION}

Formation and characterization of andoic aluminium oxide templates

Current/voltage characteristics: The electrical current passing through the apparatus was measured throughout the second anodization of the aluminium sample. A typical currenttime plot for this case is shown in Fig. 1. The current pattern, with a rapidly decreasing and pronounced minimum current, followed by a larger steady-state current. In this case, the minimum current was found to be about $38.1 \mathrm{~mA} / \mathrm{cm}^{2}$, while the steady-state current was about $60 \mathrm{~mA} / \mathrm{cm}^{2}$. It is believed the initially high current followed by its rapid descent is the result of the barrier layer formation, followed by pore nucleation and growth at the minimum current. The steady state current is caused by the oxide growth rate at the metal/oxide interface and the oxide etch rate at the solution/oxide interface at the pore bottoms being equal.

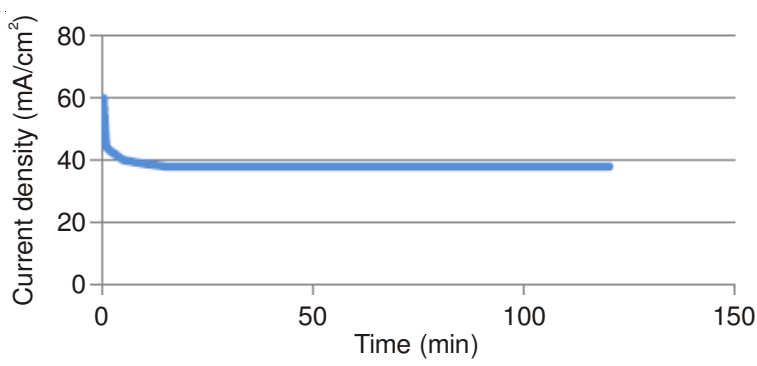

Fig. 1. The current time characteristic of anodic aluminum oxide film anodized in $20 \% \mathrm{H}_{3} \mathrm{PO}_{4}, 5^{\circ} \mathrm{C}, 60 \mathrm{~V}$

Atomic force microscope images: Anodic aluminium oxide template were characterized using atomic force microscope, with particular attention to the morphology of their top surfaces. (Fig. 2) shows a micrograph of the top surface of a anodic aluminum oxide template produced from aluminum foil under an anodization voltage of $60 \mathrm{~V}$. The resulting pores are fairly uniform in shape and diameter, narrow pore size distribution recorded by the same atomic force microscope test and the average diameter is $130 \mathrm{~nm}$.
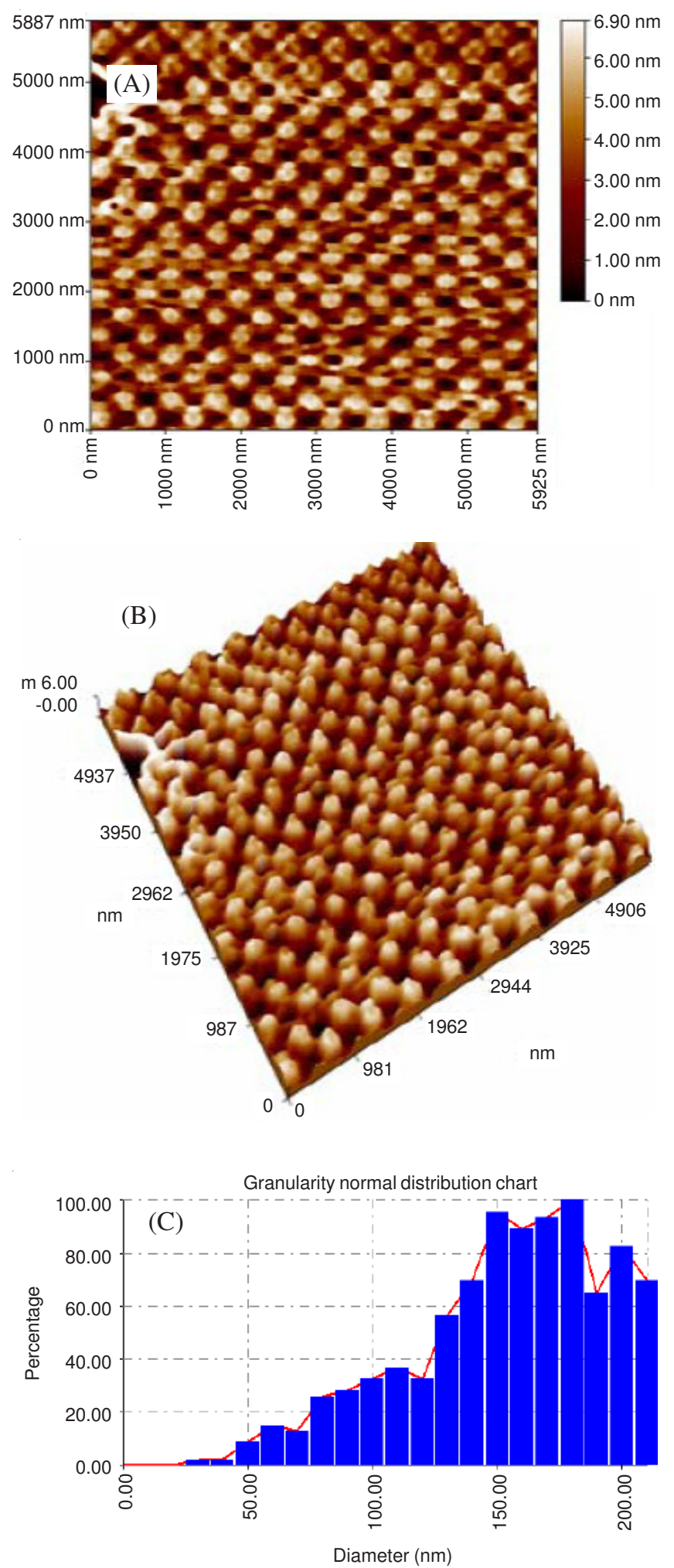

Fig. 2. Atomic force microscope topographic views (A) $2 \mathrm{D}$ and (B) 3D, (C) normal pore size distribution chart of AAO fabricated in $20 \%$ Phosphoric acid at $5^{\circ} \mathrm{C}$ for $2 \mathrm{~h}$ 
Structural characterization: Fig. 3 shows the XRD spectra of anodic aluminium oxide which was obtained in phosphoric acid at $60 \mathrm{~V}$; the change in the FCC to Monoclinic structure is noticeable at 111 and 200 plane, which correspond to 38.9 and 45.1 on the $2^{\circ}$. The low broad peak at 38.9 and 45.1 might be indicative of amorphous aluminum oxide compound formed ${ }^{10}$. Where a peak of a maximum intensity corresponding to aluminium base metal appeared at this due to large pore diameter $200 \mathrm{~nm}$.

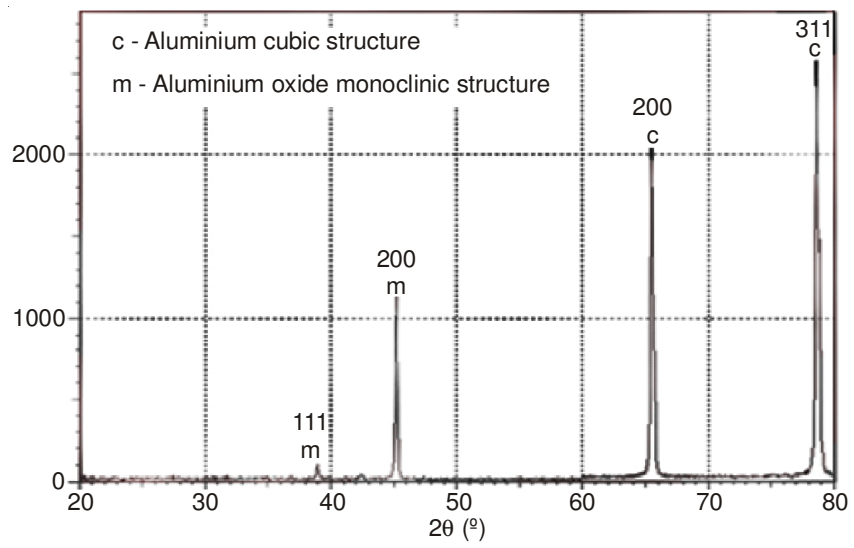

Fig. 3. XRD patterns of aluminum after anodizing for $2 \mathrm{~h}$ at $60 \mathrm{~V}$ in $5 \%$ phosphoric acid electrolyte at $5{ }^{\circ} \mathrm{C}$

\section{Platinum nanowires fabrication}

FTIR, UV-vissible and atomic absorbtion spectroscopy: Platinum-phenothaiozine complexes was freshly prepared to be used as a fuel to fabricate the Pt nanowire via electrodeposition procedure, FTIR, UV-vissible and atomic absorbtion spectroscopy were conducted to examine the electronic structures of this complex.the results revealed the following suggested structures:

$\left[\mathrm{PtL}_{2} \mathrm{Cl}_{4}\right] . \mathrm{H}_{2} \mathrm{O}$.(tetra chloro-bis-[10- $H$-dibenzo-

[b,e]-1,4-thiazine] Platinum(IV). Hydrate).

As expected, FT-IR gave good information about the complex behaviour of the ligand with various metal ion. The IR spectra of the free ligands (phenothiazine) have also been registered; characteristic frequencies of the C-N (at $1469 \mathrm{~cm}^{-1}$ ), C-S (at $655 \mathrm{~cm}^{-1}$ ). The IR spectra of the ligand also exhibited band at $3340 \mathrm{~cm}^{-1}$ and assigned to symmetrical stretching vibration for $(\mathrm{N}-\mathrm{H})$ group, while the bending vibration appeared at $1597 \mathrm{~cm}^{-1}$. The $\mathrm{C}-\mathrm{H}$ group asymmetrical stretching vibration appeared at $2924 \mathrm{~cm}^{-1}$, while the symmetrical stretching vibration appeared at $2854 \mathrm{~cm}$. The $\mathrm{C}=\mathrm{C}$ group appeared at $1570 \mathrm{~cm}^{-1}$. The most important conclusions from the IR spectra of the isolated complex was: N-H band disappeared in IR spectra of the of the platinum-phenothiozine complex and accured the shift in another groups weak band stretching vibration of coordinate of metal-nitrogen appeared at $455 \mathrm{~cm}$, this result is very probable a consequence of $\mathrm{L}$ coordination as a monodentate ligand through nitrogen atom. A band observed around (3468-3444) $\mathrm{cm}^{-1}$ in the spectra of the complex, assigned to the $\mathrm{v}(\mathrm{OH})$, which refer to presence of $\mathrm{H}_{2} \mathrm{O}$ uncoordination or out of sphere.

The electronic spectra of metal complex was recorded in their solution in DMF in the range 250-1100 nm at room temperature. The electronic spectrum of free ligand $(\mathrm{L})$ exhibited one main band. The absorption band appeared at $319 \mathrm{~nm}$ (31348) $\mathrm{cm}^{-1}$ due to interaligand $\left(\pi \rightarrow \pi^{*}\right)$ transition. PtL: The platinum complex is diamagnetic as expected ${ }^{11}$, the ground state suggested spin-paired octahedral stereochemistry geometry. The atomic ground state is ${ }^{5} \mathrm{D}$. Two principle spinallowed absorption bands are to be expected corresponding to the transition from the ${ }^{1} \mathrm{~A}_{1 \mathrm{~g}}$ and ${ }^{1} \mathrm{~T}_{2 \mathrm{~g}}$ excited states in addition, two bands assigned to the spin-forbidden singlet-triplet transition may be observed at lower energies than the spin allowed transition $^{11}$. The spectrum of the prepared brown platinum complex shows three bands in the visible region assigned to the ${ }^{1} \mathrm{~A}_{1 \mathrm{~g}} \rightarrow{ }^{1} \mathrm{~T}_{1 \mathrm{~g}},{ }^{1} \mathrm{~A}_{1 \mathrm{~g}} \rightarrow{ }^{1} \mathrm{~T}_{2 \mathrm{~g}}, \mathrm{~L} \rightarrow \mathrm{PtCT}$ transitions. The transition observed at (10928.96) $\mathrm{cm}^{-1}$ is assigned to the spin-forbidden transition ${ }^{1} \mathrm{~A}_{1 \mathrm{~g}} \rightarrow{ }^{3} \mathrm{~T}_{1 \mathrm{~g}}{ }^{12,13}$.

Atomic force microscope analysis of Pt nanowire: Atomic force microscope micrograph of Pt nanowire is shown in Fig. 4. We can find out that the abundant and free defect nanowires have been prepared. The diameter of the nanowires is about $136 \mathrm{~nm}$ and approximately equal to those of the nanopores of anodic aluminum oxide template used in our experiments. The particle size distribution shown in (Fig. 4c).

SEM analysis of Pt nanowire array electrode: Fig. 5 a and $b$ are the top views of the Pt nanowires at lower and higher magnifications respectively. The electrochemical plating was carried out for (10 min). According to the SEM images, the average diameter of the wire is $(38 \mathrm{~nm})$ and the distance from the

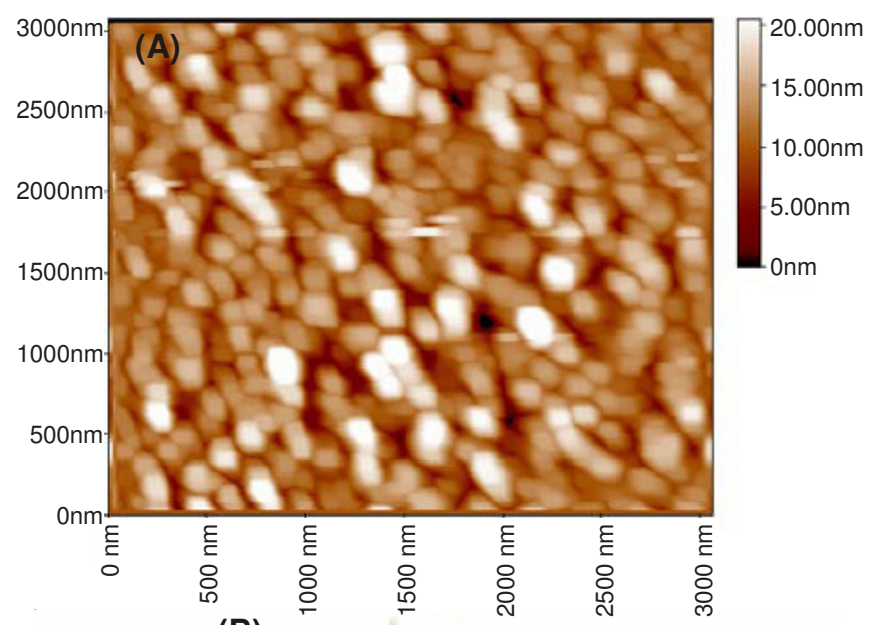

(B)

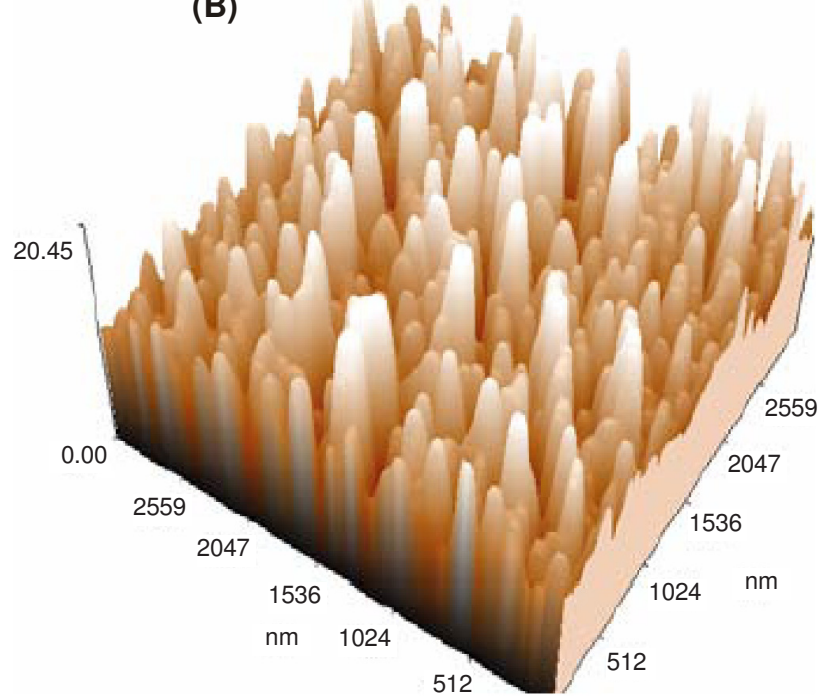




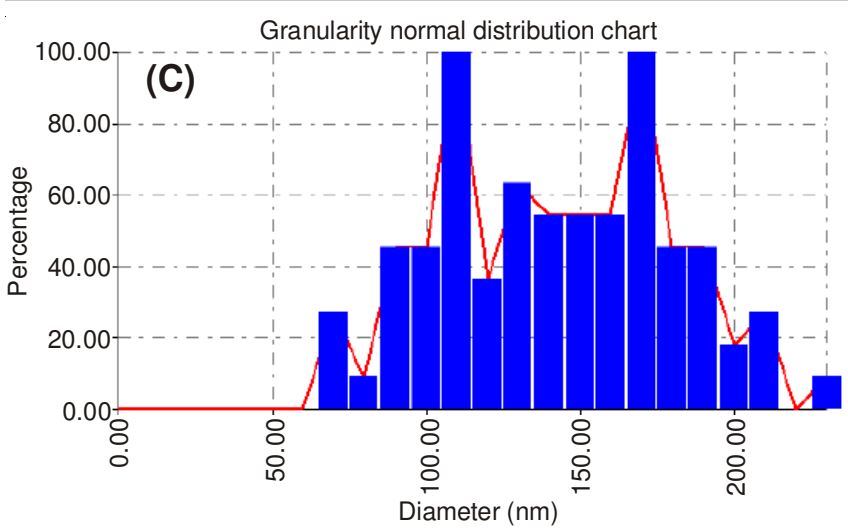

Fig. 4. Atomic force microscope top image of platinum nanowires array grown on the surface of an anodic aluminum oxide membrane by using electrodeposition methods: (A) 2D, (B) 3D, (C) particle size distribution of grown Pt nanowires

center of the wire to the center of the neighboring wire is $(3 \mathrm{~nm})$. They are consistent with the pore size and the interpore distance of the template. It is noticed that the Pt nanowires have about the same height and nearly flat top surfaces.
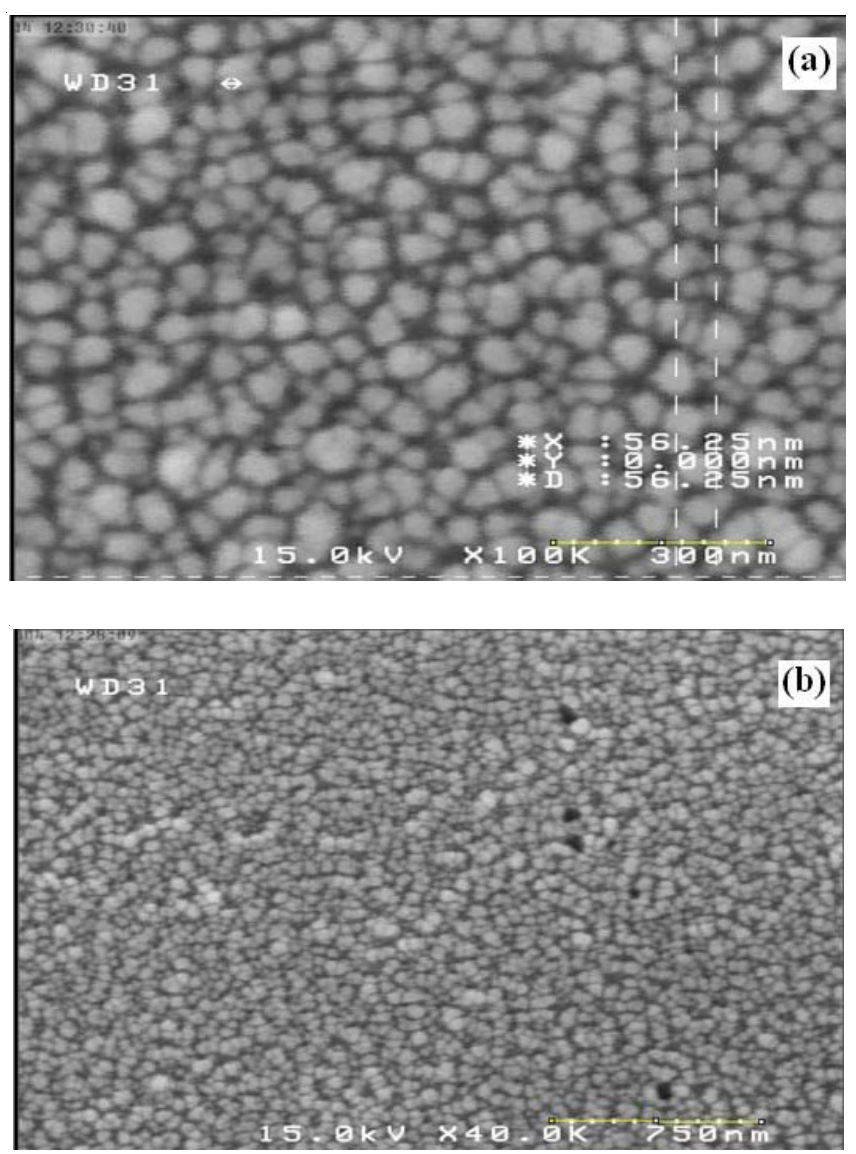

Fig. 5. SEM images of Pt nanowires synthesized with the anodic aluminum oxide template

XRD analysis of Pt nanowire arrays: The crystalline nature of the Pt nanowire was studied by powder X-ray diffraction. Fig. 6 shows the XRD diffractograms of Pt depo- sition in anodic aluminum oxide membrane. This sample is all highly $\mathrm{Pt}(111)$ oriented. The Pt has a face-centered cubic structure. Here, the metal element $\mathrm{Pt}$ is deposited on $\mathrm{Al}_{2} \mathrm{O}_{3}$ substrates. Because metal elements possess higher surface free energy (e.g., $\mathrm{Pt}(111)$ is $3290 \mathrm{erg} / \mathrm{cm}^{2}$ than the $\mathrm{Al}_{2} \mathrm{O}_{3}$ substrates.

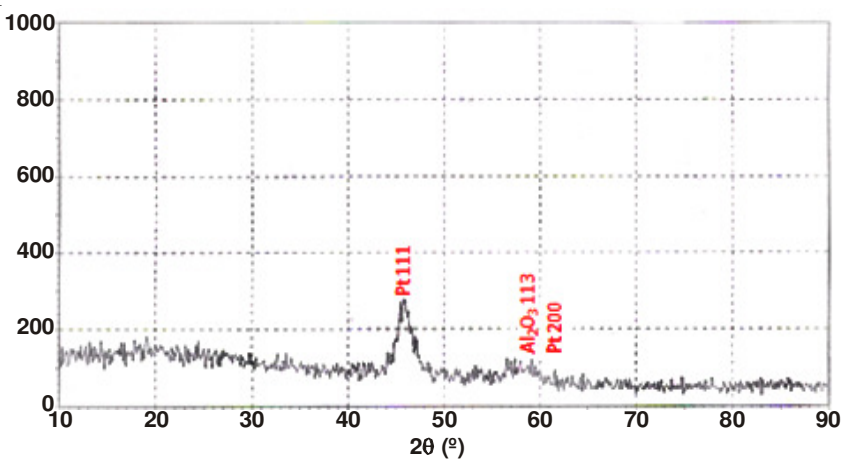

Fig. 6. XRD spectra of platinum nanowires prepared in anodic aluminum oxide membrane. The measurement was performed without removing the anodic aluminum oxide membranes

\section{Conclusion}

It has been demonstrated that the electrodeposition of platinum metal from phenathizine complex within a porous anodic aluminum oxide template yields highly ordered Ptnanowires. This electrodeposition approach offers the obvious advantage that the deposition of particles starts at the aluminum cathode on the bottom of the nanopores and the length of Ptwires can be easily controlled by the deposition time, We believe that this approach offers a new and convenient route to fabricate metal Pt-wires arrays and may find potential application in fuel cell and sensing devices and so on.

\section{REFERENCES}

1. L. Zaraska, G.D. Sulka and M. Jaskula, J. Phys., 146, 12 (2009).

2. D. Lo and R.A. Budiman, J. Electrochem. Soc., 154, 60 (2007).

3. N. Tasaltin, S. Öztürk, S.H. Yüzer and Z.Z. Öztürk, J. Optoelect. Biomed. Mater, 1, 79 (2009).

4. X.-Z. Gong, J.-N. Tang, J.-Q. Li and Y.-K. Liang, Trans. Nonferrous Met. Soc. China, 18, 642 (2008).

5. J. Fang, P. Spizzirri, L. Lin, A. Roberts and S. Prawer, J. Australian Ceramic Soc., 46, 46 (2010).

6. L. Lee and Y. Kwon, Hard Templates for Fabrication of Nanostructured Films, NANO: Brief Reports and Reviews, 5, 75 (2010).

7. H.D. Yan, P. Lemmens, H. Dierke, S.C. White, F. Ludwig and M. Schilling, J. Phys.: Conf. Series, 145, Article ID 012079 (2009).

8. Z.F. Zhou, Y.C. Zhou, Y. Pan and X.G. Wang, Mater. Lett., 62, 3419 (2008).

9. G. Cao and D. Liu, Adv. Colloid Interf. Sci., 136, 45 (2008).

10. A.O. Araoyinbo, A.F.M. Noor, S. Sreekantan and A. Aziz, Int. J. Mechanical Mater. Eng., 5, 53 (2010).

11. E.I. Solomon and A.B.P. Lever, Inorganic Electronic Structure and Spectroscopy: Applications and Case Studies, John Wiley \& Sons Inc, New York, Chester, Singapore, Toronto, vol. 2 (2006).

12. B.N. Figgis and M.A. Hitchman, Ligand Field Theory and its Application, Wiley-VCH, New York, Singapore, Toronto (2000).

13. N.N. Greenwood and A. Ernshaw, Chemistry Elements, Pergamum Press, Inc., edn. 2 (1998). 